\title{
Distinguishing two kinds of scalar mesons from heavy meson decays
}

\author{
Wei Wang ${ }^{* a, b}$ and Cai-Dian Lü ${ }^{\dagger} a$ \\ a Institute of High Energy Physics, Chinese Academy of Sciences, Beijing 100049, People's Republic of China \\ ${ }^{b}$ Istituto Nazionale di Fisica Nucleare, Sezione di Bari, Bari 70126, Italy
}

(Dated: November 7, 2018)

\begin{abstract}
In the $\mathrm{SU}(3)$ symmetry limit, semileptonic $D^{+} \rightarrow S l^{+} \nu$ and $B^{-} \rightarrow S l^{-} \bar{\nu}$ decays, with $S=a_{0}(980)$, $f_{0}(980)$ and $f_{0}(600)$, are found to obey different sum rules in the $\bar{q} q$ and the tetra-quark descriptions for scalar mesons. Thus these sum rules can distinguish the two scenarios for light scalar mesons model-independently. This method also applies to the $\bar{B}^{0} \rightarrow J / \psi\left(\eta_{c}\right) S$ decays. Two kinds of SU(3) symmetry breaking effects are found to be under control, which will not spoil our method. The branching fractions of the $D^{+} \rightarrow S l^{+} \nu, B^{-} \rightarrow S l^{-} \bar{\nu}$ and $\bar{B}^{0} \rightarrow J / \psi\left(\eta_{c}\right) S$ decays roughly have the order $10^{-4}, 10^{-5}$ and $10^{-6}$, respectively. The ongoing BES-III and the forthcoming Super B experiments are able to measure these channels and accordingly to provide detailed information of the scalar meson inner structure.
\end{abstract}

PACS numbers: 13.20.Fc; 13.20.He; $13.25 . \mathrm{Hw} ; 14.40 . \mathrm{Cs}$

\section{INTRODUCTION}

Understanding the internal structure of scalar mesons is of prime interest in hadron physics for several decades. It plays a crucial role to understand the chiral symmetry breaking mechanisms of the QCD and the confinement of hadrons. In spite of the striking success of QCD theory for strong interaction the underlying structure of the light scalar mesons is still under controversy [1 5$]$. The classification of scalar mesons suffers from large hadronic uncertainties arising from the fact that scalar mesons share the same spin-parity quantum numbers $J^{P C}=0^{++}$with the QCD vacuum. For instance irrespective of the dispute on the existence of $f_{0}(600)$ and $K_{0}^{*}(800)$ mesons, scalar mesons have been identified as ordinary $\bar{q} q$ states, four-quark states or meson-meson bound states or even those supplemented with a scalar glueball. In reality, the nonperturbative QCD fluctuations induce the mixing between different content, which will add further complexities.

At present, there are already many experimental studies on the production of scalar mesons in nonleptonic $D$ decays. For instance branching ratios (BRs) of $D^{+} \rightarrow f_{0}(600) \pi^{+}$and $D^{+} \rightarrow f_{0}(980) \pi^{+}$have the order of $10^{-3}$ and $10^{-4}$, respectively [6]. On the theoretical side nonleptonic $D$ decays receive large hadronic uncertainties, which can hinder us from getting a clear view of the internal structures of scalar mesons. On the contrary semileptonic $\mathrm{D}^{+} \rightarrow \mathrm{Sl}^{+} \nu$ decays only contain one scalar meson in the final state, which can be better candidates to probe different structure scenarios of scalar mesons.

In this work, we propose a model-independent way to distinguish two different descriptions for scalar mesons, i.e. the two-quark and the four-quark scenarios, through the semileptonic $B^{-} \rightarrow S l \bar{\nu}$ and/or $D^{+} \rightarrow S l^{+} \nu$ decays, where $S$ denotes a scalar meson among $a_{0}(980), f_{0}(980)$ and $f_{0}(600)$. In the following method the flavor SU(3) symmetry will be used to derive different sum rules for the BRs under these two scenarios. These semileptonic heavy meson decays are clean as they do not receive much pollution from the hadronic interactions like nonleptonic heavy meson decays. In $B$ decays, the lepton pair can also be replaced by a charmonium state since they own the same properties in the flavor $\mathrm{SU}(3)$ space. For instance, the mode $\bar{B}^{0} \rightarrow J / \psi S$ is probably much easier for the experiments to observe.

The layout of this work is given as follows. In Sec. [II we will briefly discuss different descriptions for scalar mesons. The SU(3) relations for the productions rates and the symmetry breaking effects are discussed in Sec. III and Sec. IV. respectively. The last section contains a couple of remarks and our conclusion.

\footnotetext{
* Email: wwang@ihep.ac.cn

$\dagger$ Email: lucd@ihep.ac.cn
} 


\section{TWO SCENARIOS FOR SCALAR MESONS}

A number of scalar mesons have been experimentally discovered in different processes, however in contrast to the pseudoscalar and vecor mesons the identification of scalar mesons is more difficult because of their large decay widths causing a strong overlap between different resonances and background. In particular, in recent years there has been controversies about the existence of the two light and very broad states: $K_{0}^{*}(800)$ (also refereed as $\kappa$ ) meson in the $700-900 \mathrm{MeV}$ region; $f_{0}(600)$ (also denoted as $\sigma$ ) meson in the region of $400-1200 \mathrm{MeV}$.

The $f_{0}(600)$ meson, having the same quantum number with the QCD vacuum, might play a significant role in the chiral symmetry breaking and the mass origin of the pseudoscalar mesons. This meson might also have some relation with the scalar meson proposed in the linear sigma model at 50 years ago 7]. There have been many studies on the possible scalar resonance structure in different experimental processes. Although the data of the $2 \pi$ invariant mass spectra in the $p \bar{p}$ annihilation do not show a distinct resonance structure below $900 \mathrm{MeV}[8]$, the existence of the $f_{0}(600)$ meson is allowed in many processes for instance the $\pi N$ scattering [9], the nonleptonic $D^{+} \rightarrow \pi^{+} \pi^{-} \pi^{+}$ decay channel [10 12], and the $J / \psi \rightarrow \omega \pi^{+} \pi^{-}$[13] and $\psi(2 S) \pi^{+} \pi^{-}$[14, 15]. This pole is also derived in the analysis based on chiral symmetry and Roy equations [16, 17] and the analysis using unitarized chiral perturbation theory [18]. Refs. [19, 20] analyzed the $\sigma \rightarrow \gamma \gamma$ process and found that the data are consistent with a two-step process of $\gamma \gamma \rightarrow \pi^{+} \pi^{-}$with a subsequent final state interaction $\pi^{+} \pi^{-} \rightarrow \pi^{0} \pi^{0}$. This conclusion prevents us from learning anything new from the coupling of $f_{0}(600)$ with $2 \gamma$. This situation is also similar for the $K_{0}^{*}(800)$ meson. For example, the data from the BES-II implies a $\kappa$-like structure in $J / \psi$ decays into $\bar{K}^{0 *} K^{+} \pi^{-}$[21, 22]. A number of phenomenological analysis find a light and broad state consistent with $K_{0}^{*}(800)$ (see many references in the review [1] in the PDG [6]) but this pole is absent in some other work [23 26]. The inconsistence between different analysis implies the complexities in the nature of the $f_{0}(600)$ and $K_{0}^{*}(800)$ resonances.

Assuming the existence of the $K_{0}^{*}(800)$ and $f_{0}(600)$, there are 9 mesons together below or near $1 \mathrm{GeV}$, and in this case it is reasonable to assume the nonet for scalar mesons below or near $1 \mathrm{GeV}$ consisting of $f_{0}(600), K_{0}^{*}(800), f_{0}(980)$ and $a_{0}(980)$. In the $\bar{q} q$ picture, scalar mesons are viewed as P-wave states [27], whose flavor wave functions are given by

$$
\begin{aligned}
& \left|f_{0}(600)\right\rangle=\frac{1}{\sqrt{2}}(|\bar{u} u\rangle+|\bar{d} d\rangle) \equiv|\bar{n} n\rangle, \quad\left|f_{0}(980)\right\rangle=|\bar{s} s\rangle, \\
& \left|a_{0}^{0}(980)\right\rangle=\frac{1}{\sqrt{2}}(|\bar{u} u\rangle-|\bar{d} d\rangle), \quad\left|a_{0}^{-}(980)\right\rangle=|\bar{u} d\rangle, \quad\left|a_{0}^{+}(980)\right\rangle=|\bar{d} u\rangle .
\end{aligned}
$$

In this picture, $f_{0}(980)$ is mainly made up of $\bar{s} s$, which is supported by the large production rates in $J / \psi \rightarrow \phi f_{0}(980)$ and $\phi \rightarrow f_{0}(980) \gamma$ decays [6]. Meanwhile, the experimental data also indicates the nonstrange component of $f_{0}(980)$ : the $\mathrm{BR}$ of $J / \psi \rightarrow \omega f_{0}(980)$ is comparable with that of $J / \psi \rightarrow \phi f_{0}(980)$. To accommodate with the experimental data, $f_{0}(980)$ is supposed to be the mixture of $\bar{n} n$ and $\bar{s} s$ as

$$
\begin{aligned}
\left|f_{0}(980)\right\rangle & =|\bar{s} s\rangle \cos \theta+|\bar{n} n\rangle \sin \theta, \\
\left|f_{0}(600)\right\rangle & =-|\bar{s} s\rangle \sin \theta+|\bar{n} n\rangle \cos \theta .
\end{aligned}
$$

Using the BRs of $J / \psi \rightarrow f_{0}(980) \phi$ and $J / \psi \rightarrow f_{0}(980) \omega$, the ratio between the coupling constants of $f_{0}(980) K \bar{K}$ and $f_{0}(980) \pi \pi$, and the BR of $\phi \rightarrow f_{0}(980) \gamma$, the mixing angle $\theta$ is constrained as [28]

$$
25^{\circ}<\theta<40^{\circ}, \quad 140^{\circ}<\theta<165^{\circ} .
$$

Due to the large decay width of $f_{0}(600)$ meson, the mixing angle is usually identified as an energy dependent variable. In the above determination, the mixing angle has been taken as a constant variable.

From the allowed range of the mixing angle given above, we can see that $f_{0}(980)$ is dominated by the $\bar{s} s$ component. The expected dominant decay channel $f_{0}(980) \rightarrow K \bar{K}$ is suppressed by the small phase space. Then $f_{0}(980) \rightarrow \pi \pi$ decay becomes dominant arising from some nonperturbative interactions such as the rescattering mechanism. This is supported by the experimental data [6]. 
The classical $\bar{q} q$ picture meets with several difficulties. For example, since $s$ quark is expected to be heavier than $u / d$ quark, it is difficult to explain the fact that the strange meson $K_{0}^{*}(800)$ is lighter than the isotriplet mesons $a_{0}(980)$, and the isosinglet meson $f_{0}(980)$ has a degenerate mass with $a_{0}(980)$. Moreover, since scalar mesons are identified as the $\mathrm{P}$-wave states in the $\bar{q} q$ description, it is difficult to explain why the $K_{0}^{*}(800)$ meson is lighter than its vector partner $K^{*}(892)$.

Inspired by these difficulties, other candidate scenarios are proposed. In Ref. [29], scalar mesons are identified as diquark-diquark states. In the $\mathrm{SU}(3)$ flavor space, the two quarks can form two multiplets as

$$
3 \otimes 3=\overline{3} \oplus 6,
$$

while the other two antiquarks reside in 3 or $\overline{6}$ multiplets. The diquark in a scalar meson is taken to be totally antisymmetric for all quantum numbers, color antitriplet, flavor antitriplet, spin 0 . The $q^{2}(\bar{q})^{2}$ states make a flavor nonet, whose internal structures are given as:

$$
\begin{aligned}
& \left|f_{0}(600)\right\rangle=|\bar{u} u \bar{d} d\rangle, \quad\left|f_{0}(980)\right\rangle=|\bar{n} n \bar{s} s\rangle, \\
& \left|a_{0}^{0}(980)\right\rangle=\frac{1}{\sqrt{2}}|(\bar{u} u-\bar{d} d) \bar{s} s\rangle, \quad\left|a_{0}^{+}(980)\right\rangle=|\bar{d} u \bar{s} s\rangle, \quad\left|a_{0}^{-}(980)\right\rangle=|\bar{u} d \bar{s} s\rangle .
\end{aligned}
$$

Taking the mixing into account, the isosinglet mesons are expressed as

$$
\begin{aligned}
\left|f_{0}(980)\right\rangle & =|\bar{n} n \bar{s} s\rangle \cos \phi+|\bar{u} u \bar{d} d\rangle \sin \phi, \\
\left|f_{0}(600)\right\rangle & =-|\bar{n} n \bar{s} s\rangle \sin \phi+|\bar{u} u \bar{d} d\rangle \cos \phi,
\end{aligned}
$$

where the $\phi$ is constrained as $[\underline{30}]$

$$
\phi=\left(174.6_{-3.2}^{+3.4}\right)^{\circ}
$$

Apart from the $\bar{q} q$ and the tetraquark picture (diquark-diquark), there exists another promising candidate interpretation of scalar mesons: they are molecule states. Ref. 31] found within a potential model that scalar tetraquark systems can only appear in the form of the $K \bar{K}$ molecules. The pair of the isodoublet kaons can form four different states: three mesons as an isovector and one isosinglet meson, which can easily explain the degeneracy of the mass of $a_{0}(980)$ and $f_{0}(980)$. More importantly the $K_{0}^{*}(800)$ and $f_{0}(600)$ mesons are irrelevant and thus this scheme is also consistent with the absence of these two resonances as physical states. The molecule description has also been successfully applied in different processes for instance the effective Hamiltonian approach within the molecule picture has been used to compute the $f_{0}(980) \rightarrow \pi \pi$ and $f_{0}(980) \rightarrow \gamma \gamma$ decays [32]. It is worthwhile mentioning that our proposed method in the following section is invalid under this interesting picture since the production rates of $f_{0}(600)$ will be used.

The identification of the scalar mesons below $1 \mathrm{GeV}$ as the four-quark states or molecule states can raise the question about the scalar $\bar{q} q$ states. The experimentalists have observed several scalar mesons above $1 \mathrm{GeV}$. The expectation from the naive quark model that $\bar{q} q$ scalar mesons $(L=1)$ are expected to be heavier than the vector $\bar{q} q$ partners $(L=0)$ may also give us a hint that the scalar $\bar{q} q$ nonet is made of the several heavier mesons: the isovector and isodoublet mesons $a_{0}(1450), K_{0}^{*}(1430)$, the isosinglet mesons $f_{0}(1370), f_{0}(1500), f_{0}(1710)$ with some ambiguities in the choice of the ninth member since the isosinglet mesons can mix with the scalar gluebll state through the QCD interactions. Nevertheless, since this work mainly concerns the scalar mesons below $1 \mathrm{GeV}$, we will refrain from more discussions of the nature of this heavier $\bar{q} q$ nonet.

\section{SU(3) RELATIONS}

Although the nature of scalar mesons is very complicated, we will focus on two simple pictures and analyze their productions in the heavy meson decays. Feynman diagrams for $D^{+} \rightarrow S l^{+} \nu$ decays in two different pictures are given in Fig. 1. The left panel is for the two-quark scenario, while the right panel is for the four-quark scenario. If a 


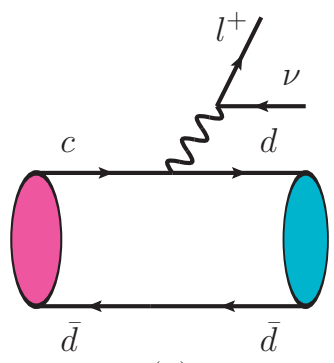

(a)

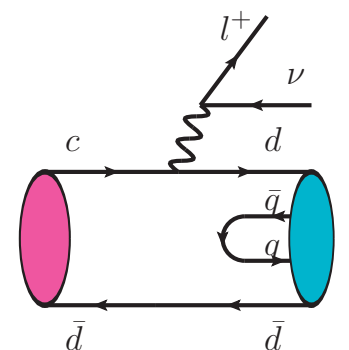

(b)

FIG. 1: Feynman diagrams of $D^{+}$decays into a scalar meson. The left diagram is for two-quark picture, while the right diagram is for the four-quark mesons.

scalar meson is made of $\bar{q} q$, the light quark is generated from the electroweak vertex and the antiquark $\bar{d}$ serves as a spectator. Thus only the component $\bar{d} d$ contributes to semileptonic $D$ decays. In the SU(3) symmetry limit, decay amplitudes of $D \rightarrow f_{0}(980)\left(f_{0}(600)\right) l \nu$ channels under the $q \bar{q}$ picture have the following relation

$$
\begin{aligned}
& \mathcal{A}\left(D^{+} \rightarrow f_{0}(980) l^{+} \nu\right)=-\sin \theta \hat{\mathcal{A}}, \\
& \mathcal{A}\left(D^{+} \rightarrow f_{0}(600) l^{+} \nu\right)=-\cos \theta \hat{\mathcal{A}},
\end{aligned}
$$

where the transition amplitude $\hat{\mathcal{A}}$ is defined as

$$
\hat{\mathcal{A}} \equiv \mathcal{A}\left(D^{+} \rightarrow a_{0}^{0}(980) l^{+} \nu\right) .
$$

This leads to a sum rule

$$
\mathcal{B}\left(D^{+} \rightarrow a_{0}^{0}(980) l^{+} \nu\right)=\mathcal{B}\left(D^{+} \rightarrow f_{0}(980) l^{+} \nu\right)+\mathcal{B}\left(D^{+} \rightarrow f_{0}(600) l^{+} \nu\right),
$$

which is independent of the mixing angles. One may worry about the accuracy of our above result because of the possible large QCD scattering effect. However, if we use the hadron picture, we can still get the same result. The $d \bar{d}$ pair produced from the weak interaction in Fig 1 (a) can form isospin 0 and isospin 1 states with the ratio of 1:1. The ratio is directly obtained from the Clebsch-Gordan coefficients. Although the QCD scattering can mix between states, the non-perturbative QCD interactions conserve the isospin. Therefore the sum of production rates of isospin 0 states on the right hand side of eq.(10) is always equal to production rates of the isospin 1 states on the left hand side of eq. (10). The isospin breaking effect in strong interaction is negligible.

If a scalar meson is composed of four quarks, besides the light quark from the electroweak vertex and the spectator, another $\bar{q} q$ pair is generated from the QCD vacuum. This quark pair could be $\bar{s} s$ or $\bar{u} u$, where both $\bar{d} d \bar{s} s$ and $\bar{u} u \bar{d} d$ contribute. The decay amplitudes are given as

$$
\begin{aligned}
& \mathcal{A}\left(D^{+} \rightarrow f_{0}(980) l^{+} \nu\right)=-(\cos \phi+\sqrt{2} \sin \phi) \hat{\mathcal{A}}, \\
& \mathcal{A}\left(D^{+} \rightarrow f_{0}(600) l^{+} \nu\right)=(\sin \phi-\sqrt{2} \cos \phi) \hat{\mathcal{A}},
\end{aligned}
$$

which gives

$$
\mathcal{B}\left(D^{+} \rightarrow a_{0}^{0}(980) l^{+} \nu\right)=\frac{1}{3}\left[\mathcal{B}\left(D^{+} \rightarrow f_{0}(980) l^{+} \nu\right)+\mathcal{B}\left(D^{+} \rightarrow f_{0}(600) l^{+} \nu\right)\right]
$$

It is meaningful to define the ratio of partial decay widths

$$
R=\frac{\mathcal{B}\left(D^{+} \rightarrow f_{0}(980) l^{+} \nu\right)+\mathcal{B}\left(D^{+} \rightarrow f_{0}(600) l^{+} \nu\right)}{\mathcal{B}\left(D^{+} \rightarrow a_{0}^{0}(980) l^{+} \nu\right)} .
$$


The ratio is 1 for the two-quark description, while it is 3 for the four-quark description of scalar mesons. Similarly for semileptonic $B \rightarrow S l \bar{\nu}$ decays, the charm quark in Fig 1 is replaced by a bottom quark and the $\bar{d}$ quark is replaced by a $\bar{u}$ quark, while leptons are replaced by their charge conjugates. We have the same sum rules

$$
\begin{aligned}
R & =\frac{\mathcal{B}\left(B^{-} \rightarrow f_{0}(980) l^{-} \bar{\nu}\right)+\mathcal{B}\left(B^{-} \rightarrow f_{0}(600) l^{-} \bar{\nu}\right)}{\mathcal{B}\left(B^{-} \rightarrow a_{0}^{0}(980) l^{-} \bar{\nu}\right)} \\
& =\left\{\begin{array}{l}
1 \text { two quark } \\
3 \text { tetra-quark }
\end{array}\right.
\end{aligned}
$$

Let's now examine whether all these channels are experimentally measurable by estimating the branching ratios for individual channels. If the mixing angle is close to $\theta=0^{\circ}$ or $\theta=90^{\circ}$ in the two-quark picture (in four-quark scenario, the mixing angle is $54.7^{\circ}$ or $\left.144.7^{\circ}\right)$, either $f_{0}(600)$ or $f_{0}(980)$ meson has small production rates but the other one should have large production rates. Neglecting the highly suppressed channel, the ratio defined in eq.(13 14) can still distinguish the two different scenarios for scalar mesons.

If the mixing angle is not close to the values discussed in the above paragraph, all three $D^{+} \rightarrow S l^{+} \nu$ channels would have similar BRs in magnitude. The BR of the semileptonic $D_{s} \rightarrow f_{0}(980)$ decay is measured [33] as

$$
\begin{aligned}
& \mathcal{B}\left(D_{s} \rightarrow f_{0}(980) l \bar{\nu}\right) \times \mathcal{B}\left(f_{0}(980) \rightarrow \pi^{+} \pi^{-}\right) \\
& =(2.0 \pm 0.3 \pm 0.1) \times 10^{-3}
\end{aligned}
$$

Thus as an estimation, generic BRs for the cascade $D^{+} \rightarrow S l^{+} \nu$ decays are expected to have the order

$$
f(D)=\frac{V_{c d}^{2}}{V_{c s}^{2}} \times 2 \times 10^{-3} \simeq 1 \times 10^{-4},
$$

while the mixing effects can modify the BRs by several times. For instance if the mixing angles given in Eq. (3) and Eq. (7) are used, one has the estimates of the BRs

$$
\begin{aligned}
& \mathcal{B}\left(D^{+} \rightarrow f_{0}(980) l^{+} \nu\right) \simeq \frac{1}{2} f(D) \tan ^{2} \theta \simeq(0.04-0.35) \times 10^{-4} \\
& \mathcal{B}\left(D^{+} \rightarrow a_{0} l^{+} \nu\right) \simeq \frac{1}{2} f(D) \frac{1}{\cos ^{2} \theta} \simeq(0.6-0.8) \times 10^{-4} \\
& \mathcal{B}\left(D^{+} \rightarrow f_{0}(600) l^{+} \nu\right) \simeq \frac{1}{2} f(D) \simeq(0.4-0.6) \times 10^{-4}
\end{aligned}
$$

in two-quark description; or

$$
\begin{aligned}
& \mathcal{B}\left(D^{+} \rightarrow f_{0}(980) l^{+} \nu\right) \simeq(0.19-0.63) \times 10^{-4}, \\
& \mathcal{B}\left(D^{+} \rightarrow a_{0} l^{+} \nu\right) \simeq(0.5-0.54) \times 10^{-4}, \\
& \mathcal{B}\left(D^{+} \rightarrow f_{0}(600) l^{+} \nu\right) \simeq(0.88-1.4) \times 10^{-4}
\end{aligned}
$$

in the four-quark description, where the mixing angle given in Eq. (7) is used but the uncertainty is increased as $(175 \pm 10)^{\circ}$. The luminosity of BES-III experiment at BEPC II in Beijing is designed as $3 \times 10^{32} \mathrm{~cm}^{-2} \mathrm{~s}^{-1}$. This experiment, starting running since summer 2008, will accumulate 30 million $D \bar{D}$ pair per running year [34]. Even we assume the detect efficiency is only $20 \%$, there will be 600 events per running year if the BR is used as $1 \times 10^{-4}$. It is very likely to observe these decay channels.

As for the $B$ decays, the generic BR of $B \rightarrow S l \bar{\nu}$ can be estimated utilizing the $B \rightarrow \rho l \bar{\nu}$ and $D_{s}^{+} \rightarrow \phi l^{+} \nu$ decays

$$
\begin{aligned}
f_{1}(B)=\mathcal{B}(B \rightarrow S l \bar{\nu}) & \simeq \mathcal{B}(B \rightarrow \rho l \bar{\nu}) \frac{\mathcal{B}\left(D_{s} \rightarrow f_{0}(980) l \bar{\nu}\right)}{\mathcal{B}\left(D_{s} \rightarrow \phi l \bar{\nu}\right)} \\
& \simeq 10^{-4} \times \frac{10^{-3}}{10^{-2}}=10^{-5}
\end{aligned}
$$

where the heavy quark symmetry has been assumed. The mixing effects can be similarly analyzed as in the case of semileptonic $D$ decays. For the three distinct channels, the BRs are obtained from Eqs. (17) and (18) by changing 


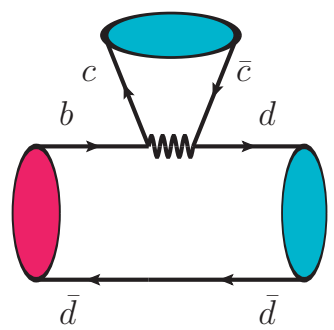

(a)

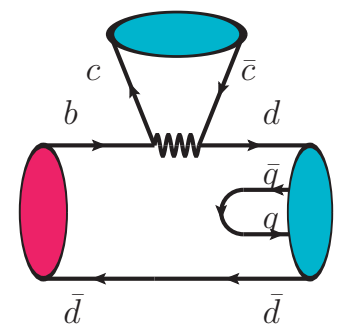

(b)

FIG. 2: Feynman diagrams of $B \rightarrow J / \psi\left(\eta_{c}\right) S$ decays. The left panel is for two-quark picture, while the right panel is for the four-quark mesons.

$f(D)$ by $f(B)$ and changing $10^{-4}$ by $10^{-5}$. It is also worthwhile pointing out that the above analysis is based on the heavy quark limit $m_{b, c} \rightarrow \infty$. The finite heavy quark masses effect might also provide some changes. Compared with the recently measured semileptonic $B \rightarrow \eta$ decay [35]

$$
\mathcal{B}\left(B^{-} \rightarrow \eta l^{-} \bar{\nu}\right)=(3.1 \pm 0.6 \pm 0.8) \times 10^{-5},
$$

we can see that the $B \rightarrow S l \bar{\nu}$ is comparable with the $B \rightarrow \eta l \bar{\nu}$ decays. Such a large BR offers a great opportunity for distinguishing the descriptions. Even if the present $B$ factory does not observe these channels, it is easy for the forthcoming Super B factory to measure these channels.

The semileptonic $D / B$ decays are clean, which do not receive much pollution from the strong interaction. But since the neutrino is identified as missing energy, the efficiency to detect these channels may be limited. The lepton pair can also be replaced by some other $\mathrm{SU}(3)$ singlet systems such as a $J / \psi$ or $\eta_{c}$ meson. Replacing the lepton pair by the $J / \psi$ and replacing $B^{-}$by a $\bar{B}^{0}$ state (a different spectator antiquark will not change the results) in Eq. (14), one can easily obtain the similar sum rules for the BRs

$$
\begin{aligned}
R & =\frac{\mathcal{B}\left(\bar{B}^{0} \rightarrow f_{0}(980) J / \psi\right)+\mathcal{B}\left(\bar{B}^{0} \rightarrow f_{0}(600) J / \psi\right)}{\mathcal{B}\left(\bar{B}^{0} \rightarrow a_{0}^{0}(980) J / \psi\right)} \\
& = \begin{cases}1 & \text { two quark } \\
3 & \text { tetra-quark }\end{cases}
\end{aligned}
$$

The Feynman diagrams are shown in Fig. 2. Although these channels are hadronic decays, the hadronic uncertainties are mostly canceled in the sum rule of ratios. The BR is expected to have the order

$$
\begin{aligned}
f^{\prime}(B)=\mathcal{B}(B \rightarrow S J / \psi) & \simeq \mathcal{B}\left(\bar{B}^{0} \rightarrow \rho^{0} J / \psi\right) \frac{\mathcal{B}\left(D_{s} \rightarrow f_{0}(980) l \bar{\nu}\right)}{\mathcal{B}\left(D_{s} \rightarrow \phi l \bar{\nu}\right)} \\
& \simeq 2.7 \times 10^{-5} \times \frac{10^{-3}}{10^{-2}} \simeq 3 \times 10^{-6} .
\end{aligned}
$$

The BRs for the three $B \rightarrow S J / \psi$ decay channels can be obtained by the results given in Eqs. (17) and (18) with a multiplication of the factor 0.03 . On experimental side the $J / \psi$ is easily detected through a lepton pair $l^{+} l^{-}$and thus these modes may be more useful. If the $J / \psi$ meson is replaced by $\eta_{c}$ in eq.(21), one can get the similar sum rules.

With the available data in the future, one can not only distinguish the different descriptions for scalar mesons but also determine the mixing angle between the isosinglet mesons. In Fig. 3, we show the dependence of ratios of BRs in $D / B$ decays on the mixing angle in both scenarios. For all three kinds of decays, the solid (black) and dashed (blue) lines represent ratios $R_{f_{0}(980)}=\frac{\mathcal{B}\left(D / B \rightarrow f_{0}(980) l \nu\right)}{\mathcal{B}\left(D / B \rightarrow a_{0}^{0} l \nu\right)}\left(R_{f_{0}(980)}=\frac{\mathcal{B}\left(B \rightarrow f_{0}(980) J / \psi\right)}{\mathcal{B}\left(B \rightarrow a_{0}^{0} J / \psi\right)}\right)$ in the $\bar{q} q$ and $q^{2} \bar{q}^{2}$ picture, respectively. Similarly, we can define the ratio $R_{f_{0}(600)}=\frac{\mathcal{B}\left(D / B \rightarrow f_{0}(600) l \nu\right)}{\mathcal{B}\left(D / B \rightarrow a_{0}^{0} l \nu\right)}\left(R_{f_{0}(600)}=\frac{\mathcal{B}\left(B \rightarrow f_{0}(600) J / \psi\right)}{\mathcal{B}\left(B \rightarrow a_{0}^{0} J / \psi\right)}\right)$. Unfortunately, due to the large uncertainty of the $f_{0}(600)$ meson mass as we will discuss in the following section, we have to modify the ratio by a phase space factor $\kappa$ for channels involving $f_{0}(600)$, especially for the semileptonic $D$ decays. In Fig [3, the dotted (red) and dot-dashed (green) lines represent ratios $\kappa R_{f_{0}(600)}$ in the $\bar{q} q$ and $q^{2} \bar{q}^{2}$ picture, respectively. The mixing angles in either scenario are clearly related to the branching ratios. 


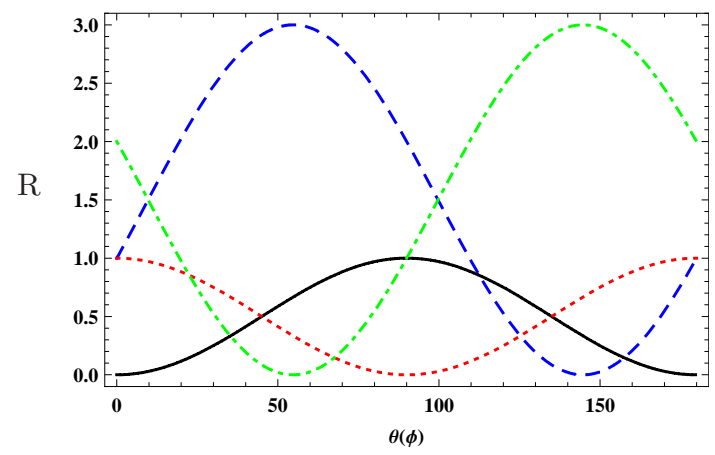

FIG. 3: Ratios of branching fractions in $D / B$ decays vs mixing angles in two-quark and four-quark scenarios. For all three kinds of decays, the solid (black) and dashed (blue) lines represent ratios $R_{f_{0}(980)}$ in the $\bar{q} q$ and $q^{2} \bar{q}^{2}$ picture, respectively. The dotted (red) and dot-dashed (green) lines represent ratios $\kappa R_{f_{0}(600)}$ in the $\bar{q} q$ and $q^{2} \bar{q}^{2}$ picture, respectively.

\section{SU(3) SYMMETRY BREAKING}

Remember that the above results are obtained in the flavor SU(3) symmetry limit. It is also necessary to estimate the symmetry breaking effects since the strange quark is heavier than $u, d$ quark. In the following we will first analyze the generic size of symmetry breaking effects and then discuss two different cases.

The large SU(3) symmetry breaking effects in $D$ meson decays, for example the BR of $D^{0} \rightarrow K^{+} K^{-}$is about three times larger than $D^{0} \rightarrow \pi^{+} \pi^{-}$, may lead to the suspect of the reliability of the sum rules as in Eq. (14). However the hadronic $D^{0} \rightarrow K^{+} K^{-}$and $D^{0} \rightarrow \pi^{+} \pi^{-}$decays are different with the decay modes discussed in the this work in several aspects. The former decays involve two light mesons and the symmetry breaking effects coming from either of them will be constructive ${ }^{1}$. In semileptonic $B / D$ decay modes the final state contains only one hadron and the symmetry breaking is naturally smaller. Moreover in hadronic $D$ decays, the $D \rightarrow K / \pi$ transitions is induced by the different quark current $c \rightarrow s / d$. On the contrary, the transition current at the quark level in this work is $c \rightarrow d$, same for channels involving isovector and isosinglet scalar mesons. Accordingly the symmetry breaking effects in semileptonic $D / B$ decays will be smaller than those in hadronic $D$ decays.

Generically the size of the $\mathrm{SU}(3)$ breaking effect could be roughly estimated by the mass difference between $u / d$ and $s$ quarks, whose magnitude is

$$
\frac{m_{s}-m_{d / u}}{\Lambda} \sim 0.3
$$

where $\Lambda$ is the hadronic scale. Taking into this generic symmetry breaking effect, the two ratios become $R=1 \pm 0.3$ in the $\bar{q} q$ picture and $R=3 \pm 0.9$ in the tetraquark picture. The difference between them could be smaller, but they are still different and our method is still useful. We also expect smaller SU(3) symmetry breaking effects in $B$ meson decays, since the large energy release may weaken the effects from the different masses.

One particular origin is that the isospin singlet scalar mesons have different masses, which can change the phase space in the semileptonic $D / B$ decays. Fortunately, this $\mathrm{SU}(3)$ breaking effect can be well studied, which almost does not depend on the internal structure of scalar mesons or the strong interactions. The mass of $f_{0}(980)$ is well measured but the mass of $f_{0}(600)$ meson has large uncertainties $m_{f_{0}(600)}=(0.4-1.2) \mathrm{GeV}$. This big range of masses indeed induces large differences to $D$ decays, since the $D$ meson mass is only $1.87 \mathrm{GeV}$. The $\mathrm{BR}$ of the semileptonic decay is affected by a factor of $(0.31-5.4)$ depending on the mass of the $f_{0}(600)$ meson. Therefore the sum rule in eq. (13) is

\footnotetext{
1 It is clearer if the factorization method is used although the conclusion does not depend on this hypothesis. The symmetry breaking is either from the different $D \rightarrow K$ and $D \rightarrow \pi$ form factors or decay constants of kaon and pion. In $D^{0} \rightarrow K^{+} K^{-}$both form factors and decay constants are larger.
} 
not good unless the $f_{0}(600)$ meson mass is well measured. But in $B$ meson decays, the sum rule in eq.(14) will not be affected sizably, since the $f_{0}(600)$ meson mass is negligible compared with the large $B$ meson mass. Numerically, this correction factor in $B$ decays is $(0.9-1.1)$. This will also affect the extraction of the mixing angle as we have discussed in the previous section.

Another SU(3) breaking effect comes from the decay form factors of various scalar mesons. In the two-quark scenario, only the $\bar{d} d$ component contributes to the transition form factors shown in the left diagram of Fig.1. The $\mathrm{SU}(3)$ symmetry breaking effect to the form factors is thus negligible. In the four-quark scenario, the $\bar{u} u \bar{d} d$ component in $f_{0}(980)$ and $f_{0}(600)$ resonance is different from the internal structure of $a_{0}^{0}: \frac{1}{\sqrt{2}}(\bar{u} u-\bar{d} d) \bar{s} s$. In the right diagram of Fig 1 it would be easier to produce the lighter $\bar{u} u$ (or $\bar{d} d$ ) quark from the vacuum than the $\bar{s} s$ quark. The $\mathrm{SU}(3)$ symmetry breaking effects may make the form factor of $D / B \rightarrow f_{0}(600)$ and $D / B \rightarrow f_{0}(980)$ larger than that of $D / B \rightarrow a_{0}$. It will make the ratio $R$ larger than 3 in the four-quark scenario. Thus this $\mathrm{SU}(3)$ symmetry breaking effects in the form factors will not spoil our method but instead it will improve its applicability.

\section{DISCUSSIONS AND CONCLUSION}

In the above two sections, we have discussed the SU(3) relations for the BRs and the symmetry breaking effects in the heavy meson decays. The individual production rates for $f_{0}(600)$ and $f_{0}(980)$ are required in our method. The effect in the phase space factor caused by the large uncertainties in the mass of the $f_{0}(600)$ can be directly taken into account. However the physical processes would be more complicated since both the $f_{0}(600)$ and $f_{0}(980)$ need be reconstructed from the $\pi \pi$ mode ${ }^{2}$. Considering the large width of $f_{0}(600)$, it is difficult to distinguish these two mesons by the same final state. In this case, the simple ratios $R_{f_{0}(980)}$ and $\kappa R_{f_{0}(600)}$ will be not useful to constrain the mixing angle. Nevertheless, the ratio $R$ which needs only the sum of these two states can still provide a method for distinguishing the two descriptions for scalar mesons.

One more ambiguity comes from the mysterious quark content of scalar mesons. In this work, we have payed particular attention to the two-quark and tetra-quark picture. As we have mentioned in the introduction section the physical situation is more subtle, since any meson may have two- and four-quark components as part of the usual Fock state expansion. Those two-quark and four-quark states are quantum-mechanically mixed, also likely with other potential candidates with the same quantum numbers. Restricted to the 2-quark and 4-quark mixtures, two complex decay amplitudes and several mixing angles are unknown and should be treated as input parameters, while only 3 physical observables are available from the experiments in principle. This mixing problem is not solvable and the proposed sum rules becomes useless. Nevertheless our method is at least helpful to rule out one of the possibility. If the ratio $R$ were close to 1 , the pure 4 -quark picture is likely to be ruled out but if the ratio $R$ were close to 3 the pure 2-quark picture is likely to be excluded.

In conclusion, we have investigated the possibility to distinguish the two-quark and tetra-quark picture for light scalar mesons. The semileptonic $D / B \rightarrow S l \bar{\nu}$ decays and the nonleptonic $B \rightarrow J / \psi\left(\eta_{c}\right) S$ decays are discussed in detail. These decay channels have a large potential to be measured on the ongoing BES-III and the forthcoming Super B experiments. Despite a number of ambiguities arising from the nonperturbative QCD dynamics or the SU(3) symmetry breaking effects, our method is useful to distinguish these two different scenarios.

\footnotetext{
$2 \rho$ is a vector meson and its decay into $\pi \pi$ occur via the $P$-wave amplitude, while the decay amplitude of $f_{0}(600)$ and $f_{0}(980)$ into $\pi \pi$ belongs to $S$-wave. The background from the $\rho^{0} \rightarrow \pi \pi$ can be separated within the partial wave analysis. Another possibility is to use only the $\pi^{0} \pi^{0}$ mode in the analysis, since $\rho^{0}$ can not decay to this final state due to the "wrong" C parity. Of course, we need more data in this case.
} 


\section{Acknowledgement}

W. Wang would like to thank Pietro Colangelo for useful discussions. This work is partially supported by National Natural Science Foundation of China under the Grant No. 10735080, 10625525 and 10805037 and Natural Science Foundation of Zhejiang Province of China, Grant No. Y606252.

[1] S. Spanier, N. A. Tornqvist and C. Amsler, "Note on Scalar Mesons," Review published on Particle Data Group.

[2] S. Godfrey and J. Napolitano, Rev. Mod. Phys. 71, 1411 (1999).

[3] F. E. Close and N. A. Tornqvist, J. Phys. G 28, R249 (2002).

[4] R. L. Jaffe, Phys. Rept. 409, 1 (2005) [Nucl. Phys. Proc. Suppl. 142, 343 (2005)] arXiv:hep-ph/0409065.

[5] M. R. Pennington, Prog. Theor. Phys. Suppl. 168, 143 (2007) arXiv:hep-ph/0703256.

[6] C. Amsler et al. [Particle Data Group], Phys. Lett. B 667, 1 (2008).

[7] Y. Nambu, Phys. Rev. Lett. 4 (1960) 380; Y. Nambu and G. Jona-Lasinio, Phys. Rev. 122, 345 (1961); Y. Nambu and G. Jona-Lasinio, Phys. Rev. 124, 246 (1961); M. Gell-Mann and M. Levy, Nuovo Cim. 16 (1960) 705.

[8] C. Amsler et al. [Crystal Barrel Collaboration], Phys. Lett. B 355 (1995) 425; A. Abele et al. [Crystal Barrel Collaboration], Phys. Lett. B 380 (1996) 453; Phys. Lett. B 397, 350 (1997).

[9] R. Kaminski, L. Lesniak and K. Rybicki, Z. Phys. C 74, 79 (1997) arXiv:hep-ph/9606362.

[10] E. M. Aitala et al. [E791 Collaboration], Phys. Rev. Lett. 86, 770 (2001) arXiv:hep-ex/0007028.

[11] J. M. Link et al. [FOCUS Collaboration], Phys. Lett. B 585, 200 (2004) arXiv:hep-ex/0312040.

[12] G. Bonvicini et al. [CLEO Collaboration], Phys. Rev. D 76, 012001 (2007) arXiv:0704.3954 [hep-ex]].

[13] M. Ablikim et al. [BES Collaboration], Phys. Lett. B 598, 149 (2004) arXiv:hep-ex/0406038.

[14] A. Gallegos, J. L. Lucio M. and J. Pestieau, Phys. Rev. D 69, 074033 (2004) arXiv:hep-ph/0311133.

[15] M. Ablikim et al. [BES Collaboration], Phys. Lett. B 645, 19 (2007) arXiv:hep-ex/0610023.

[16] G. Colangelo, J. Gasser and H. Leutwyler, Nucl. Phys. B 603, 125 (2001) arXiv:hep-ph/0103088.

[17] I. Caprini, G. Colangelo and H. Leutwyler, Phys. Rev. Lett. 96, 132001 (2006) arXiv:hep-ph/0512364.

[18] I. Caprini, Phys. Rev. D 77, 114019 (2008) arXiv:0804.3504 [hep-ph]].

[19] M. R. Pennington, Phys. Rev. Lett. 97, 011601 (2006).

[20] M. R. Pennington, Mod. Phys. Lett. A 22, 1439 (2007) arXiv:0705.3314 [hep-ph]].

[21] M. Ablikim et al. [BES Collaboration], Phys. Lett. B 633, 681 (2006) arXiv:hep-ex/0506055.

[22] F. K. Guo, R. G. Ping, P. N. Shen, H. C. Chiang and B. S. Zou, Nucl. Phys. A 773, 78 (2006) arXiv:hep-ph/0509050.

[23] S. N. Cherry and M. R. Pennington, Nucl. Phys. A 688, 823 (2001) arXiv:hep-ph/0005208.

[24] S. Kopp et al. [CLEO Collaboration], Phys. Rev. D 63, 092001 (2001) arXiv:hep-ex/0011065.

[25] J. M. Link et al. [FOCUS Collaboration], Phys. Lett. B 621, 72 (2005) arXiv:hep-ex/0503043.

[26] B. Aubert et al. [BABAR Collaboration], Phys. Rev. D 76, 011102 (2007) arXiv:0704.3593 [hep-ex]].

[27] N. A. Tornqvist, Z. Phys. C 68, 647 (1995).

[28] H. Y. Cheng, C. K. Chua and K. C. Yang, Phys. Rev. D 73, 014017 (2006); and many references therein.

[29] R. L. Jaffe, Phys. Rev. D 15, 267 (1977); Phys. Rev. D 15, 281 (1977).

[30] L. Maiani, F. Piccinini, A. D. Polosa and V. Riquer, Phys. Rev. Lett. 93, 212002 (2004).

[31] J. D. Weinstein and N. Isgur, Phys. Rev. Lett. 48, 659 (1982);Phys. Rev. D 27, 588 (1983); Phys. Rev. D 41, 2236 (1990).

[32] T. Branz, T. Gutsche and V. E. Lyubovitskij, Eur. Phys. J. A 37, 303 (2008) arXiv:0712.0354 [hep-ph]]; T. Branz, T. Gutsche and V. E. Lyubovitskij, Phys. Rev. D 78, 114004 (2008) arXiv:0808.0705 [hep-ph]].

[33] K. M. Ecklund [CLEO Collaboration], arXiv:0907.3201 [hep-ex].

[34] D. M. Asner et al., arXiv:0809.1869 [hep-ex].

[35] B. Aubert et al. [BABAR Collaboration], arXiv: 0808.3524 [hep-ex]. 